\title{
Bacterial Taxa Associated with High Adherence to Mediterranean Diet in a Spanish Population ${ }^{\dagger}$
}

\author{
Carles Rosés 1,* , Amanda Cuevas-Sierra 2, Salvador Quintana ${ }^{3}$, José I. Riezu-Boj 2,4, \\ J. Alfredo Martínez ${ }^{2,4,5}$, Fermín I. Milagro ${ }^{2,4,5}$ and Anna Barceló ${ }^{1}$ \\ 1 Servei de Genòmica i Bioinformàtica, Universitat Autònoma de Barcelona, Barcelona, Spain \\ 2 Centre for Nutrition Research; Department of Nutrition, Food Sciences and Physiology, \\ University of Navarra, Pamplona, Spain \\ 3 Unaffiliated \\ 4 Navarra Institute for Health Research (IdISNA), Pamplona, Spain \\ 5 Centro de Investigación Biomédica en Red de la Fisiopatología de la Obesidad y Nutrición (CIBERobn), \\ Instituto de Salud Carlos III, Madrid, Spain \\ * Correspondence: crosespol@gmail.com \\ + Presented at the The 1st International Electronic Conference on Nutrients - Nutritional and Microbiota \\ Effects on Chronic Disease, 2-15 November 2020; Available online: https://iecn2020.sciforum.net/. \\ Published: 30 October 2020
}

\begin{abstract}
The Mediterranean diet (MD) is recognized as one of the healthiest diets worldwide and is associated with the prevention of cardiovascular and metabolic diseases, among others. Dietary habits are considered one of the strongest modulators of the gut microbiota, which seems to play a significant role in the health and disease of the host. The purpose of the present study was to evaluate interactive associations between gut microbiota composition and habitual dietary intake in 360 Spanish adults of the Obekit cohort (normal weight, overweight and obese subjects). Dietary intake and adherence to the MD tests together with fecal samples were collected from each subject. Fecal $16 \mathrm{~S}$ rRNA sequencing was performed and checked against the dietary habits. MetagenomeSeq was the statistical tool applied to analyse at species taxonomic level. Results from this study confirm that a strong adherence to the MD increases the population of some beneficial bacteria improving microbiota status towards a healthier pattern. Bifidobacterium animalis is the species with the strongest association with the MD. One of the highlights is the positive association between several SCFA-producing bacteria and high adherence to MD. In conclusion, this study shows that MD, fibre, legumes, vegetables, fruit and nuts intake are associated with an increase in butyrate-producing taxa like Roseburia faecis, Ruminococcus bromii and Oscillospira (Flavonifractor) plautii.
\end{abstract}

Keywords: Bifidobacterium animalis; gut microbiota; short-chain fatty acids; obesity; butyrate

\section{Introduction}

The gut microbiota status has a direct impact on health and disease of the host [1]. Dietary habits are considered one of the strongest modulators of the gut microbiota. Serious conditions can showup due to sedentarism and bad dietary habits: hypertrophied adipocytes release inflammatory molecules (i.e., interleukins and tumour necrosis factor) which over enough time can favour the development of several inflammation-related disorders such as metabolic syndrome, cardiovascular disease, colorectal cancer, neurodegenerative diseases [2,3] and autoimmune disorders like Crohn's disease, ulcerative colitis and allergies [4]. In this context, the Mediterranean diet (MD) is recognized as one of the healthiest diets worldwide. Therefore, we would expect a modulation of the gut microbiota as one of the positive health effects of the MD [5]. The main objective of the present work relays on the bacteria that are more closely associated with a high adherence to the MD. 


\section{Material and Methods}

\subsection{Subjects}

This cross-sectional study enrolled 360 Spanish adults (251 females and 109 males) ranged 45.0 \pm 10.5 years old. Major exclusion criteria included a history of diabetes mellitus, cardiovascular disease and hypertension, pregnant or lactating women, and current use of lipid-lowering drugs.

\subsection{Anthropometric and Biochemical Measurements}

The volunteers were classified as normal weight when BMI: $18.5-24.9 \mathrm{~kg} / \mathrm{m}^{2}(\mathrm{n}=64)$, overweight when BMI: $25.0-29.9 \mathrm{~kg} / \mathrm{m}^{2}(\mathrm{n}=115)$, and obesity when BMI $>30.0 \mathrm{~kg} / \mathrm{m}^{2}(\mathrm{n}=181)$. Blood biochemistry (glucose, total cholesterol (TC), high-density lipoprotein cholesterol (HDL), and triglycerides) was analysed. Insulin resistance índex (HOMA-IR) was calculated.

\subsection{Dietary Estimation}

Habitual dietary intake at baseline was collected with a validated food frequency questionnaire [6]. A 14-item questionnaire, PREDIMED validated test, was also used in this study adherence of participants to the MD [7].

\subsection{Faecal Sample Collection and DNA Extraction}

\subsubsection{Metagenomic Data: Library Preparation}

Metagenomics studies were performed by analysing the variable regions V3-V4 of the prokaryotic $16 \mathrm{~S}$ ribosomal RNA gene (16S rRNA), which gives $460 \mathrm{bp}$ amplicons in a two rounds PCR protocol. Finally, paired-end sequencing was performed in a MiSeq platform (Illumina).

\subsubsection{Metagenomics Data: Analysis and Processing}

$16 \mathrm{~S}$ rRNA sequences obtained were filtered following quality criteria of the OTU (Operational Taxonomic Units) processing pipeline LotuS (release 1.58) [8]. Taxonomy was assigned using HITdb achieving up to species-level sensitivity. BLAST was used when HITdb failed to reach an homology higher than $97 \%[9,10]$. Global normalization was performed using the library size as a correcting factor and $\log 2$ data transformation [11].

\subsection{Statistical Analysis}

Microbiome Analyst tool [12] was used for statistical differences in microbiota profiles between groups (tertiles) through Zero-inflated Gaussian approach of MetagenomeSeq and using the cumulative sum scaling (CSS) normalization.

\section{Results}

\section{Microbiota Composition: MD Adherence}

MD tertiles 1 and 3 have been compared through metagenomeSeq analysis. Significant differences appeared when comparing both tertiles (FDR $<0.05$ ). Species shown in Figure 1 are strongly influenced by the MD-score. Subjects with a higher adherence to the MD are represented in the 3rd tertile while those who are far from the MD model are in the 1st tertile. This work focus on the high adherence species and their distribution, with box plots (Figure 2). All box plots represent those species with significant differences between high and low adherence tertiles. 


\begin{tabular}{|c|c|c|c|}
\hline \multicolumn{2}{|l|}{ High adherence (3rd tertile) } & \multicolumn{2}{|l|}{ Low adherence (1st tertile) } \\
\hline Species & FDR & Species & FDR \\
\hline Bifidobacterium animalis & $1.21 \mathrm{E}-07$ & $\begin{array}{c}\text { OTU100|NN=Eubacterium saphenum } \\
\text { GU427005 } \mid D=91\end{array}$ & 4.44E-05 \\
\hline Bacteroides cellulosilyticus & 4.47E-07 & $\begin{array}{c}\text { OTU375|NN= Succinivibrio dextrinosolvens } \\
\qquad 17600 \mid D=97\end{array}$ & 0.0001 \\
\hline OTU946|NN=Paraprevotella clara $A B 331896 \mid D=86.8$ & $1.72 \mathrm{E}-05$ & $\begin{array}{c}\text { OTU759|NN= Gordonibacter pamelaeae } \\
A B 566419 \mid D=87.6\end{array}$ & 0.0005 \\
\hline $\begin{array}{c}\text { OTU1682|NN=Oscillibacter valericigenes } \\
A B 238598 \mid D=91.1\end{array}$ & $3.42 \mathrm{E}-05$ & $\begin{array}{c}\text { OTU11 } \mid N N=\text { Butyricicoccus pullicaecorum } \\
E U 410376 \mid D=89.5\end{array}$ & 0.0002 \\
\hline $\begin{array}{c}\text { OTU1065|NN= Oscillospira (Flavonifractor) plautii } \\
\qquad 18187 \mid D=86.6\end{array}$ & $3.42 \mathrm{E}-05$ & Christensenella minuta & 0.0020 \\
\hline OTU1173|NN=Roseburia faecis $A Y 804149 \mid D=94.9$ & 0.0008 & Parabacteroides goldsteinii & 0.0073 \\
\hline $\begin{array}{c}\text { OTU1517|NN=Catabacter hongkongensis } \\
A B 671763 \mid D=87\end{array}$ & 0.0008 & $\begin{array}{c}\text { OTU1625|NN=Anaerotruncus colihominis } \\
D Q 002932 \mid D=89.9\end{array}$ & 0.0120 \\
\hline OTU1296|NN=Ruminococcus bromii $D Q 882649 \mid D=92.3$ & 0.0120 & Alistipes timonensis & 0.0155 \\
\hline Erysipelatoclostridium ramosum & 0.0176 & Prevotella corporis & 0.0192 \\
\hline $\begin{array}{c}\text { OTU521|NN=Papillibacter cinnamivorans } \\
\text { AF167711 } \mid D=89\end{array}$ & 0.0463 & & \\
\hline
\end{tabular}

Figure 1. Bacterial species with a significant relation with adnerence to the MD (FDR $<0.05)$ by metagenomeSeq test. 


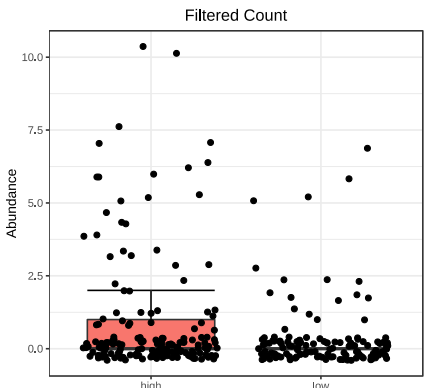

Bifidobacterium animalis

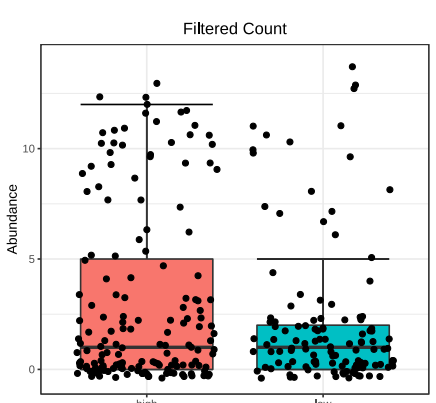

OTU1296 | NN=Ruminococcus bromi

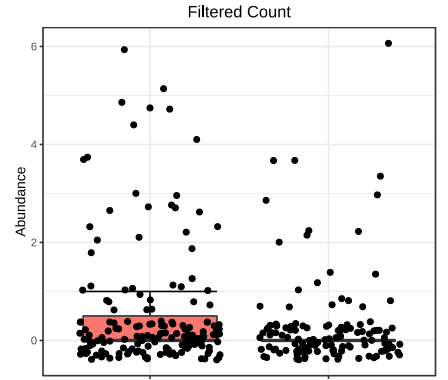

OTU1682 |NN=Oscillibacter valericigenes

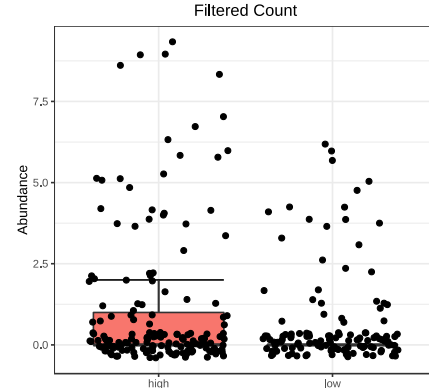

Erysipelatoclostridium ramosum

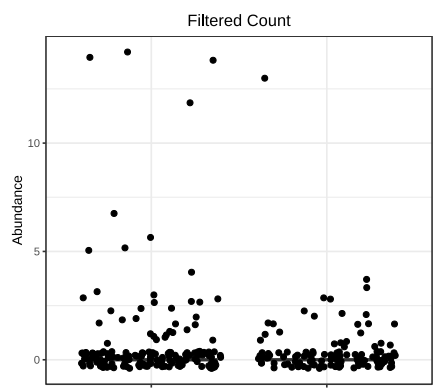

OTU946 $\mid$ NN N=Paraprevotella clara

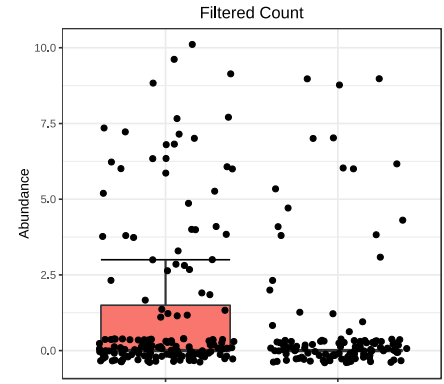

OTU1065 |NN=Oscillospira

(Flavonifractor) plautii

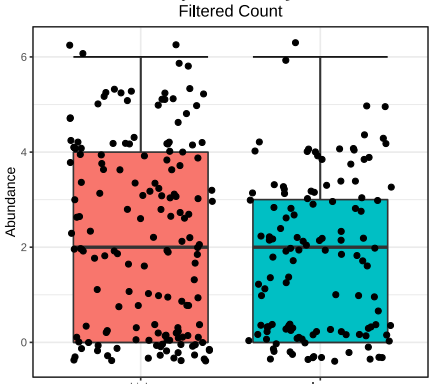

OTU521 $\mid N N=$ Papillibacter

cinnamivorans

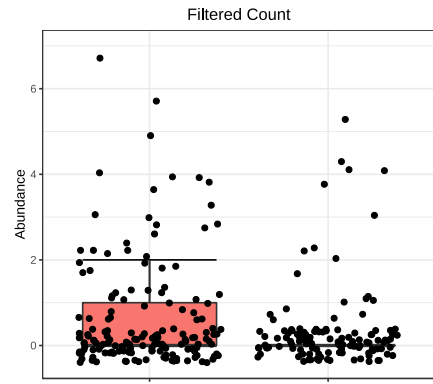

OTU1173|NN=Roseburia faecis

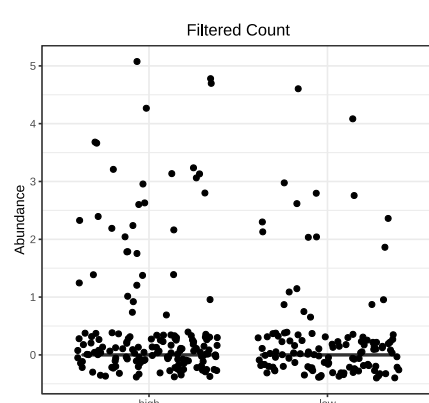

Bacteroides cellulosilyticus

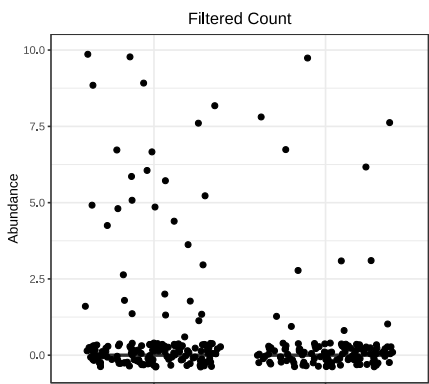

OTU1517 |NN=Catabacter hongkongensis

Figure 2. Bacterial species that were significantly more abundant in the group with high adherence to MD (FDR $<0.05)$ by metagenomeSeq test. Red boxes represent subjects with a higher adherence to the MD and blue boxes low adherence. 


\section{Discussion}

The gut microbiota co-develops with the host, and its bacterial proportions are modified by the action of the diet and other extrinsic stressors [13].

\section{High Adherence Species}

High MD adherence has many beneficial outputs to human health. It is a great resource to manage obesity-related comorbidities, such as cardiovascular diseases, type 2 diabetes and proinflammatory conditions [14-16]. Table 2 shows those species that are more associated with the adherence to MD.

Bifidobacterium animalis belongs to the pylum Bacteroides, associated with obesity-related alterations in bacterial gut microbiota and the genus Bifidobacterium might have a critical role in weight regulation [17]. B. animalis subsp. lactis GCL2508 is a probiotic strain with an anti-metabolic syndrome effect [18], capable of proliferating and producing SCFA in the gut. These compounds have a regulatory effect on inflammatory conditions [19]. Bacteroides cellulosilyticus degrade cellulose [20], with an unprecedent number of carbohydrate active enzymes providing a versatile carbohydrate utilization [21]. Paraprevotella clara, a common member of the human intestinal microbiota [22], is closely related to carbohydrate-active enzymes known to degrade insoluble fibre [23]. Indeed, P. clara is known to produce acetic acid [22]. Oscillibacter valericigenes produces valeric acid, a SCFA [24]. Valeric acid has been reported to have an inhibitory effect on histone deacetylase (HDAC) isoforms implicated in a variety of pathologies such as cancer, colitis, and cardiovascular and neurodegenerative diseases [25]. High levels of Oscillospira (Flavonifractor) plautii have been strongly correlated with a high production of SCFA, especially propionate and butyrate [26]. This species correlate with a lean host phenotype [27]. Furthermore, Oscillospira genus has been correlated with the production of secondary bile acids known to prevent Clostridium difficile-associated infectious disease in humans [28]. Roseburia faecis is a butyrate producer whose abundance has been directly related with weight loss and a reduced glucose intolerance in mice [29]. Catabacter hongkongensis, regular in the human intestinal microbiota [30] Ruminococcus bromii has been related with diets rich in fibres and resistant starch, and greatly contributes to butyrate production in the colon [31].

It is important to highlight some beneficial effects of butyric acid as it has been reported to improve the intestinal barrier integrity [32], regulate cell apoptosis [33], stimulate production of anaerobic hormones [34] and, by inducing differentiation of colonic regulatory $\mathrm{T}$ cells, suppress inflammatory and allergic responses [35]. On the other hand, many conditions have been associated with low levels of butyrate, such as colon cancer or obesity [31]; so increased butyrate production in colon may be beneficial to human health.

Erysipelatoclostridium ramosum is a member of the Erysipelotrichaceae family known to interfere in various ways with the enterohepatic circulation and excretion of bilirubin, transforming it into urobilin [36]. Papillibacter cinnamivorans is not well known but has been found in lower amount in centenaries than in any other age [37].

\section{Conclusions}

Our results indicate that the well-known beneficial factors of the MD may be triggered by changes in intestinal microbiota due to diet habits. A high adherence to MD seems to increase the abundance of some species associated with health: Bifidobacterium animalis, Oscillibacter valericigenes, Oscillospira (Flavonifractor) plautii, Roseburia faecis, Ruminococcus bromii, Butyricicoccus pullicaecorum and Papillibacter cinnamivorans. This study strongly suggests that MD increase butyrate production from R. faecis, R. Bromi and Oscillospira (Flavonifractor) plautii. Erysipelatoclostridium ramosum is the only bacteria from this study that does not show a clear beneficial effect on health, although this identification should be taken with caution. A deeper taxonomy is required to put some light into it.

Author Contributions: Conceptualization, A.B. and F.I.M.; methodology, J.I.R.-B., F.I.M., S.Q. and A.B.; formal analysis, C.R. and A.C.-S.; data curation, C.R. and A.C.-S.; writing-original draft preparation, C.R. and A.B.; 
writing-review and editing, F.I.M., J.I.R.-B. and J.A.M.; supervision, A.B., F.I.M. and J.A.M.; project administration, A.B., F.I.M. and J.A.M. All authors have read and agreed to the published version of the manuscript.

Funding: This research was funded by Ministerio de Ciencia e Innovación (CDTI: BIOTAGUT project), CIBERobn (CB12/03/30002) and Gobierno de Navarra: Obekit (PT024), Microbiota (PI035) and Nutribiota (00111411-2018-000040) projects.

Conflicts of Interest: The authors declare no conflict of interest. The funders had no role in the design of the study; in the collection, analyses, and interpretation of data; in the writing of the manuscript; or in the decision to publish the results.

\section{References}

1. Maslowski, K.M.; Mackay, C.R. Diet, gut microbiota and immune responses. Nat. Immunol. 2011, 12, 5-9, doi:10.1038/ni0111-5.

2. Rook, G.A.W. 99th Dahlem Conference on Infection, Inflammation and Chronic Inflammatory Disorders: Darwinian medicine and the 'hygiene' or 'old friends' hypothesis. Clin. Exp. Immunol. 2010, 160, 70-79, doi:10.1111/j.1365-2249.2010.04133.x.

3. Sofi, F.; Macchi, C.; Abbate, R.; Gensini, G.F.; Casini, A. Mediterranean diet and health status: An updated meta-analysis and a proposal for a literature-based adherence score. Public Health Nutr. 2014, 17, 2769-2782, doi:10.1017/S1368980013003169.

4. Le Chatelier, E.; Nielsen, T.; Qin, J.; Prifti, E.; Hildebrand, F.; Falony, G.; Almeida, M.; Arumugam, M.; Batto, J.-M.; Kennedy, S.; et al. Richness of human gut microbiome correlates with metabolic markers. Nature 2013, 500, 541-546, doi:10.1038/nature12506.

5. Rivière, A.; Selak, M.; Lantin, D.; Leroy, F.; De Vuyst, L. Bifidobacteria and Butyrate-Producing Colon Bacteria: Importance and Strategies for Their Stimulation in the Human Gut. Front. Microbiol. 2016, 7, 979, doi:10.3389/fmicb.2016.00979.

6. Shatenstein, B.; Nadon, S.; Godin, C.; Ferland, G. Development and Validation of a Food Frequency Questionnaire. Can. J. Diet. Pract. Res. 2005, 66, 67-75, doi:10.3148/66.2.2005.67.

7. Martínez-González, M.A.; García-Arellano, A.; Toledo, E.; Salas-Salvadó, J.; Buil-Cosiales, P.; Corella, D.; Covas, M.I.; Schröder, H.; Arós, F.; Gómez-Gracia, E.; et al. A 14-Item Mediterranean Diet Assessment Tool and Obesity Indexes among High-Risk Subjects: The PREDIMED Trial. PLoS ONE 2012, 7, e43134, doi:10.1371/journal.pone.0043134.

8. Hildebrand, F.; Tito, R.Y.; Voigt, A.; Bork, P.; Raes, J. Correction: LotuS: An efficient and user-friendly OTU processing pipeline. Microbiome 2014, 2, 37, doi:10.1186/2049-2618-2-37.

9. Altschul, S.F.; Gish, W.; Miller, W.; Myers, E.W.; Lipman, D.J. Basic local alignment search tool. J. Mol. Biol. 1990, 215, 403-410, doi:10.1016/S0022-2836(05)80360-2.

10. Ritari, J.; Salojärvi, J.; Lahti, L.; de Vos, W.M. Improved taxonomic assignment of human intestinal $16 \mathrm{~S}$ rRNA sequences by a dedicated reference database. BMC Genom. 2015, 16, 1056, doi:10.1186/s12864-0152265-y.

11. Gentleman, R.C.; Carey, V.J.; Bates, D.M.; Bolstad, B.; Dettling, M.; Dudoit, S.; Ellis, B.; Gautier, L.; Ge, Y.; Gentry, J.; et al. Bioconductor: Open software development for computational biology and bioinformatics. Genome Biol. 2004, 5, R80, doi:10.1186/gb-2004-5-10-r80.

12. Dhariwal, A.; Chong, J.; Habib, S.; King, I.L.; Agellon, L.B.; Xia, J. MicrobiomeAnalyst: A Web-Based Tool for Comprehensive Statistical, Visual and Meta-Analysis of Microbiome Data. Available online: https://academic.oup.com/nar/article-abstract/45/W1/W180/3760191 (accessed on 21 April 2020).

13. Lobionda, S.; Sittipo, P.; Kwon, H.Y.; Lee, Y.K. The role of gut microbiota in intestinal inflammation with respect to diet and extrinsic stressors. Microorganisms 2019, 7, 271.

14. Mendez, M.A.; Popkin, B.M.; Jakszyn, P.; Berenguer, A.; Tormo, M.J.; Sanchéz, M.J.; Quirós, J.R.; Pera, G.; Navarro, C.; Martinez, C.; et al. Adherence to a Mediterranean Diet Is Associated with Reduced 3-Year Incidence of Obesity. J. Nutr. 2006, 136, 2934-2938, doi:10.1093/jn/136.11.2934.

15. Lopez-Legarrea, P.; Fuller, N.R.; Zulet, M.A.; Martinez, J.A.; Caterson, I.D. The influence of Mediterranean, carbohydrate and high protein diets on gut microbiota composition in the treatment of obesity and associated inflammatory state. Asia Pac. J. Clin. Nutr. 2014, 23, 360-368.

16. AlEssa, H.B.; Malik, V.S.; Yuan, C.; Willett, W.C.; Huang, T.; Hu, F.B.; Tobias, D.K. Dietary patterns and cardiometabolic and endocrine plasma biomarkers in US women. Am. J. Clin. Nutr. 2017, 105, 432-441, 
doi:10.3945/ajcn.116.143016.

17. Million, M.; Maraninchi, M.; Henry, M.; Armougom, F.; Richet, H.; Carrieri, P.; Valero, R.; Raccah, D.; Vialettes, B.; Raoult, D. Obesity-associated gut microbiota is enriched in Lactobacillus reuteri and depleted in Bifidobacterium animalis and Methanobrevibacter smithii. Int. J. Obes. 2012, 36, 817-825, doi:10.1038/ijo.2011.153.

18. Horiuchi, H.; Kamikado, K.; Aoki, R.; Suganuma, N.; Nishijima, T.; Nakatani, A.; Kimura, I. Bifidobacterium animalis subsp. lactis GCL2505 modulates host energy metabolism via the short-chain fatty acid receptor GPR43. Sci. Rep. 2020, 10, 1-8, doi:10.1038/s41598-020-60984-6.

19. Den Besten, G.; Van Eunen, K.; Groen, A.K.; Venema, K.; Reijngoud, D.J.; Bakker, B.M. The role of shortchain fatty acids in the interplay between diet, gut microbiota, and host energy metabolism. J. Lipid Res. 2013, 54, 2325-2340.

20. Robert, C.; Chassard, C.; Lawson, P.A.; Bernalier-Donadille, A. Bacteroides cellulosilyticus sp. nov., a cellulolytic bacterium from the human gut microbial community. Int. J. Syst. Evol. Microbiol. 2007, 57, 15161520, doi:10.1099/ijs.0.64998-0.

21. McNulty, N.P.; Wu, M.; Erickson, A.R.; Pan, C.; Erickson, B.K.; Martens, E.C.; Pudlo, N.A.; Muegge, B.D.; Henrissat, B.; Hettich, R.L.; et al. Effects of Diet on Resource Utilization by a Model Human Gut Microbiota Containing Bacteroides cellulosilyticus WH2, a Symbiont with an Extensive Glycobiome. PLoS Biol. 2013, 11, e1001637, doi:10.1371/journal.pbio.1001637.

22. Morotomi, M.; Nagai, F.; Sakon, H.; Tanaka, R. Paraprevotella clara gen. nov., sp. nov. and Paraprevotella xylaniphila sp. nov., members of the family "Prevotellaceae" isolated from human faeces. Int. J. Syst. Evol. Microbiol. 2009, 59, 1895-1900, doi:10.1099/ijs.0.008169-0.

23. Flint, H.J.; Bayer, E.A.; Rincon, M.T.; Lamed, R.; White, B.A. Polysaccharide utilization by gut bacteria: Potential for new insights from genomic analysis. Nat. Rev. Microbiol. 2008, 6, 121-131.

24. Iino, T.; Mori, K.; Tanaka, K.; Suzuki, K.I.; Harayama, S. Oscillibacter valericigenes gen. nov., sp. nov., a valerate-producing anaerobic bacterium isolated from the alimentary canal of a Japanese corbicula clam. Int. J. Syst. Evol. Microbiol. 2007, 57, 1840-1845, doi:10.1099/ijs.0.64717-0.

25. Yuille, S.; Reichardt, N.; Panda, S.; Dunbar, H.; Mulder, I.E. Human gut bacteria as potent class I histone deacetylase inhibitors in vitro through production of butyric acid and valeric acid. PLoS ONE 2018, 13, e0201073, doi:10.1371/journal.pone.0201073.

26. Borrelli, L.; Coretti, L.; Dipineto, L.; Bovera, F.; Menna, F.; Chiariotti, L.; Nizza, A.; Lembo, F.; Fioretti, A. Insect-based diet, a promising nutritional source, modulates gut microbiota composition and SCFAs production in laying hens. Sci. Rep. 2017, 7, doi:10.1038/s41598-017-16560-6.

27. Kasai, C.; Sugimoto, K.; Moritani, I.; Tanaka, J.; Oya, Y.; Inoue, H.; Tameda, M.; Shiraki, K.; Ito, M.; Takei, Y.; et al. Comparison of the gut microbiota composition between obese and non-obese individuals in a Japanese population, as analyzed by terminal restriction fragment length polymorphism and nextgeneration sequencing. BMC Gastroenterol. 2015, 15, 100, doi:10.1186/s12876-015-0330-2.

28. Konikoff, T.; Gophna, U. Oscillospira: A Central, Enigmatic Component of the Human Gut Microbiota. Trends Microbiol. 2016, 24, 523-524.

29. Ryan, K.K.; Tremaroli, V.; Clemmensen, C.; Kovatcheva-Datchary, P.; Myronovych, A.; Karns, R.; WilsonPérez, H.E.; Sandoval, D.A.; Kohli, R.; Bäckhed, F.; et al. FXR is a molecular target for the effects of vertical sleeve gastrectomy. Nature 2014, 509, 183-188, doi:10.1038/nature13135.

30. Lau, S.K.P.; Mcnabb, A.; Woo, G.K.S.; Hoang, L.; Fung, A.M.Y.; Chung, L.M.W.; Woo, P.C.Y.; Yuen, K.-Y.; Hospital, M.; Kong, H. Catabacter hongkongensis gen. nov., sp. nov., Isolated from Blood Cultures of Patients from Hong Kong and Canada. J. Clin. Microbiol. 2007, 45, 395-401, doi:10.1128/JCM.01831-06.

31. Venkataraman, A.; Sieber, J.R.; Schmidt, A.W.; Waldron, C.; Theis, K.R.; Schmidt, T.M. Variable responses of human microbiomes to dietary supplementation with resistant starch. Microbiome 2016, 4, 33, doi:10.1186/s40168-016-0178-x.

32. Peng, L.; Li, Z.-R.; Green, R.S.; Holzman, I.R.; Lin, J. Butyrate Enhances the Intestinal Barrier by Facilitating Tight Junction Assembly via Activation of AMP-Activated Protein Kinase in Caco-2 Cell Monolayers. J. Nutr. 2009, 139, 1619-1625, doi:10.3945/jn.109.104638.

33. Ruemmele, F.M.; Schwartz, S.; Seidman, E.G.; Dionne, S.; Levy, E.; Lentze, M.J. Butyrate induced Caco-2 cell apoptosis is mediated via the mitochondrial pathway. Gut 2003, 52, 94-100, doi:10.1136/gut.52.1.94.

34. Mikkelsen, K.H.; Allin, K.H.; Knop, F.K. Effect of antibiotics on gut microbiota, glucose metabolism and body weight regulation: A review of the literature. Diabetes Obes. Metab. 2016, 18, 444-453. 
35. Furusawa, Y.; Obata, Y.; Fukuda, S.; Endo, T.A.; Nakato, G.; Takahashi, D.; Nakanishi, Y.; Uetake, C.; Kato, K.; Kato, T.; et al. Commensal microbe-derived butyrate induces the differentiation of colonic regulatory $\mathrm{T}$ cells. Nature 2013, 504, 446-450, doi:10.1038/nature12721.

36. Midtvedt, T.; Gustafsson, B.E. Microbial conversation of bilirubin to urobilins in vitro and in vivo. Acta Pathol. Microbiol. Scand. Sect. B Microbiol. 2009, 89B, 57-60, doi:10.1111/j.1699-0463.1981.tb00152_89B.x.

37. Biagi, E.; Nylund, L.; Candela, M.; Ostan, R.; Bucci, L.; Pini, E.; Nikkïla, J.; Monti, D.; Satokari, R.; Franceschi, C.; et al. Through ageing, and beyond: Gut microbiota and inflammatory status in seniors and centenarians. PLOS ONE 2010, 5, e10667, doi:10.1371/journal.pone.0010667.

Publisher's Note: MDPI stays neutral with regard to jurisdictional claims in published maps and institutional affiliations.

(C) 2020 by the authors. Submitted for possible open access publication under the terms and conditions of the Creative Commons Attribution (CC BY) license (http://creativecommons.org/licenses/by/4.0/). 